\title{
Low-Lying Quasiparticle States and Hidden Collective Charge Instabilities in Parent Cobaltate Superconductors
}

\author{
D. Qian, ${ }^{1}$ D. Hsieh, ${ }^{1}$ L. Wray, ${ }^{1}$ Y.-D. Chuang, ${ }^{2}$ A. Fedorov, ${ }^{2}$ D. Wu, ${ }^{3}$ J. L. Luo, ${ }^{3}$ N. L. Wang, ${ }^{3}$ \\ L. Viciu, ${ }^{4}$ R. J. Cava, ${ }^{4}$ and M.Z. Hasan ${ }^{1, *}$ \\ ${ }^{1}$ Department of Physics, Joseph Henry Laboratories, Princeton University, Princeton, New Jersey 08544, USA \\ ${ }^{2}$ Advanced Light Source, Lawrence Berkeley Laboratory, Berkeley, California 94305, USA \\ ${ }^{3}$ Institute of Physics, Chinese Academy of Sciences, Beijing 100080, China \\ ${ }^{4}$ Department of Chemistry, Princeton University, Princeton, New Jersey 08544, USA
}

(Received 27 December 2005; published 1 June 2006)

\begin{abstract}
We report a state-of-the-art photoemission (angle-resolved photoemission spectroscopy) study of high quality single crystals of the $\mathrm{Na}_{x} \mathrm{CoO}_{2}$ series focusing on the fine details of the low-energy states. The Fermi velocity is found to be small $(<0.5 \mathrm{eV} \AA)$ and only weakly anisotropic over the Fermi surface at all dopings, setting the size of the pair wave function to be on the order of 10-20 nm. In the low-doping regime, the exchange interlayer splitting vanishes and two-dimensional collective instabilities such as $120^{\circ}$-type fluctuations become kinematically allowed. Our results suggest that the unusually small Fermi velocity and the unique symmetry of kinematic instabilities distinguish cobaltates from most other oxide superconductors.
\end{abstract}

DOI: 10.1103/PhysRevLett.96.216405

Research on strongly correlated electron systems has led to the discovery of unconventional states of matter such as those realized in the high $T_{c}$ superconductors, quantum Hall systems, and low-dimensional quantum magnets. Triangular cobaltates $(\mathrm{Na} / \mathrm{K})_{x} \mathrm{CoO}_{2}$ are a novel class of correlated electron systems with a rich phase diagram. Superconductivity (near $x=1 / 3$ ) and correlated insulator behavior $(x=1 / 2)$ are observed in the low-doping regime, and enhanced thermoelectric power (near $x=2 / 3$ ) and an unusual spin-density-wave (SDW) state (beyond $x=3 / 4$ ) are seen in the high-doping regime [1-3]. None of the existing theories of cobaltates can account for the changes of electronic ground states with doping, and no systematic study of its low-energy dynamics (e.g., Fermi velocity, bilayer coupling, particle-hole instabilities, etc.) exists so far. In this Letter, we report the doping evolution of the single-particle dynamics, revealing a clear dichotomy in the low-energy electronic structure of cobaltates.

An early angle-resolved photoemission spectroscopy (ARPES) study [4] carried out on the host compound found only one Fermi surface (FS). A subsequent study by Yang et al. [5] reported a surface state with a large FS and broad bulk-representative quasiparticles due to the surface annealing process. Moreover, only one FS was observed, confirming the results in Ref. [4]. In this Letter, we report the fine details of the low-energy states which allow us to determine the dimensionality and the kinematic instabilities associated with the electronic structure as a function of doping. Such a study is made possible due to the lack of surface reconstruction (hence, no surface state) in our high quality crystals. The strong interlayer coupling we resolve in the high-doping regime provides evidence for a natural mechanism for the unusual magnetic order observed, even though the system is far away from the conventional halffilled Mott limit $(x=0)$. In contrast, fine details of the low-
PACS numbers: 71.20.Be, 71.30.+h, 73.20.At, 74.90.+n

energy behavior in the low-doping regime $(x=1 / 3)$ reveal a hidden two-dimensional particle-hole instability predicted in recent many-body theories. Moreover, the results allow us to reliably compare the low-energy parameters obtained on cobaltates with other materials classes and provide fundamental ingredients for developing a microscopic theory of these materials.

Spectroscopic measurements were performed with 30 $90 \mathrm{eV}$ photons with better than $10-25 \mathrm{meV}$ energy resolution and angular resolution better than $0.8 \%$ of the Brillouin zone at Advanced Light Source (ALS) beam lines 12.0.1 and 10.0.1, using Scienta analyzers with chamber pressures below $4 \times 10^{-11}$ torr. High quality single crystals over a wide doping range $x=0.3(\mathrm{Na}), 0.33$ $(\mathrm{Na}), 0.52(\mathrm{Na} / \mathrm{K}), 0.57(\mathrm{Na} / \mathrm{K}), 0.7(\mathrm{Na}), 0.75(\mathrm{Na})$, and $0.8(\mathrm{Na})$ were used for this study. Cleaving the samples in situ at $20 \mathrm{~K}$ (or $100 \mathrm{~K}$ ) resulted in shiny flat surfaces, characterized by diffraction to be clean and well ordered
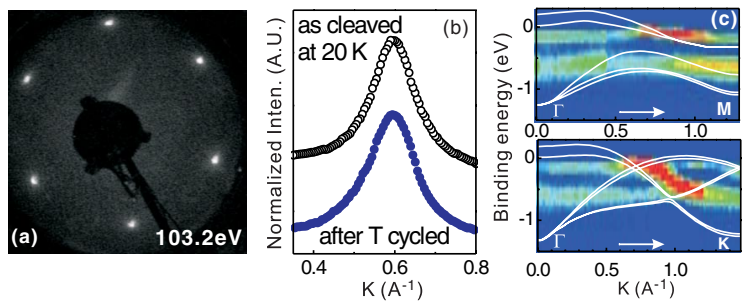

FIG. 1 (color online). (a) Low-energy electron diffraction image of the cleaved (001) surface exhibits hexagonal symmetry of the cobalt layers. No surface reconstruction is observed. (b) Temperature cycling ( $20 \mathrm{~K} \leftrightarrow 100 \mathrm{~K}$ ) was found to have no effect on the momentum distribution of the quasiparticles. Only one peak is observed at $x=0.57$ and lower dopings. (c) The valence band overlaid with band calculations [9] shows narrowing due to electron correlations. 

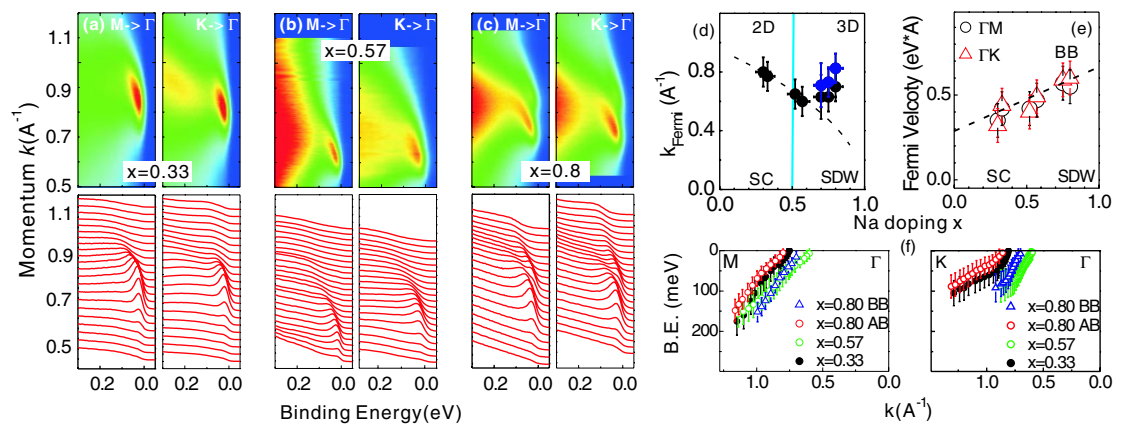

FIG. 2 (color online). Evolution of low-lying states: Single-particle removal spectra for (a) $x=0.33$, (b) $x=0.57$, and (c) $x=0.80$ along the $\Gamma-M$ and $\Gamma-K$ cuts. Low-doped samples [(a),(b)] exhibit only one Fermi crossing, while high-doped samples [(c)] show two Fermi crossings. Doping evolution of Fermi surface size $\left(k_{F}\right)$ is shown in $(\mathrm{d})$ and Fermi velocity in (e). Fermi velocity increases with increasing doping. Bonding (BB) and antibonding (AB) bands are identified at high doping. Note that the average $(\mathrm{BB}+\mathrm{AB}) \mathrm{Fermi}$ velocity decreases in approaching $x=1$. (f) Dispersion plots along the $M-\Gamma$ and $K-\Gamma$ cuts.

with the same symmetry as the bulk (Fig. 1). No surface state was observed. All presented data were taken at $20 \mathrm{~K}$, although in a few cases samples were studied at $100 \mathrm{~K}$ for cross-checking.

Figure 2 shows the doping dependence of single electron removal spectra as a function of energy and momentum. For low doping $(x<2 / 3)$, one quasiparticle feature is seen to disperse from high binding energies at high momentum values near the corner $(K)$ or the face $(M)$ of the reciprocal space to the Fermi level. High resolution study of the quasiparticle feature shows that it is well separated from the hump structure observed at $200-300 \mathrm{meV}$ binding energies at all doping. The quasiparticle lifetime drops very fast, making its intensity vanish beyond 70$100 \mathrm{meV}$ binding energies depending on the doping. The low-energy $(0-50 \mathrm{meV})$ Fermi velocities extracted from the data are shown in Fig. 2(e). The Fermi velocity is found to be weakly anisotropic (e.g., similar in magnitude along $\Gamma-M$ and $\Gamma-K$ directions) at all doping levels and increases with doping away from the Mott limit $(x=0)$. The Fermi velocity, averaged over the FS, is about $0.37 \mathrm{eV} \AA$ for $x=0.3$. Given the size of the FS, we estimate the carrier mass $m^{*} \sim \hbar k_{F} /\left|v_{F}\right| \sim 30-15 m_{e}$. This is rather large compared to most known transition metal oxide superconductors. However, a similarly large carrier mass $\left(2 m^{*} \leq 70 m_{e}\right)$ has recently been reported by $\mu \mathrm{SR}$ measurements [6]. Figure 2(d) plots the average size of the Fermi surface $\left(k_{F}\right)$ as a function of doping. Doping evolution is found to follow the 2D Luttinger theorem [FS area $\propto$ $(1-x)$ ] up to $x$ near $2 / 3$. The Fermi surface $\left(k_{F}\right)$ gets broadened and splits into two clearly separate ones beyond $x=0.75$.

Figure 3(a) shows the momentum distribution [ $n(k)$ plot] for $x=0.33$ as a representative of the low-doping regime. The inner edge of this density plot is the Fermi surface. The electron distribution in the extended zone scheme is shown in Fig. 3(b). In Fig. 4, we show the quasiparticle behavior in a typical high-doping $(x>2 / 3)$ sample. Two quasiparticles are observed to cross the Fermi level. A systematic study shows coupled oscillatory behavior of quasiparticle peak intensities with increasing incident photon energy. This is commonly observed for states with nearly orthogonal symmetries [7]. The unit cell of $\mathrm{Na}_{x} \mathrm{CoO}_{2}$ has two Co layers - therefore, the Co-derived bands with orthogonal symmetries are expected to be split, leading to two Fermi crossings similar to what is observed in multilayer materials $[7,8]$. Bilayer splitting in cobaltates has been predicted by local-density approximation (LDA) band theory [9]. Our findings are similar to the LDA prediction except that (a) the central Fermi surface is large and no corner
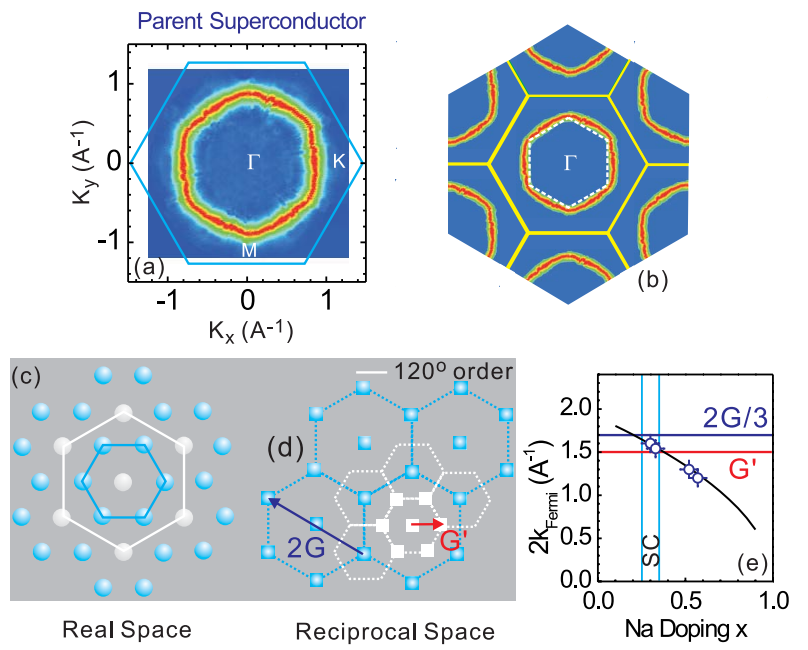

FIG. 3 (color online). Collective instabilities: (a) Momentum distribution of electrons in the $x=0.33$ sample measured in a simultaneous azimuthal scan mode with an energy window of $10 \mathrm{meV}$. (b) Electron distribution shown in an extended zone scheme. (c),(d) Real-space arrangement of cobalt atoms and $120^{\circ}$-type order and their corresponding reciprocal lattices. (e) Average electron-hole excitation wave vector $\left(2 k_{F}\right)$ as a function of Na doping $x$. In the low-doping regime, the measured data points (open circles) fall on the 2D Luttinger theorem (solid) line. Horizontal solid lines represent two commensurate instabilities. The $120^{\circ}$ order line $\left(G^{\prime}\right)$ intersects the $\left(2 k_{F}\right)$ line near $x=0.34$ and the $2 G / 3$ lattice vector line intersects near $x=0.25$, enclosing the superconducting phase boundaries [14]. 

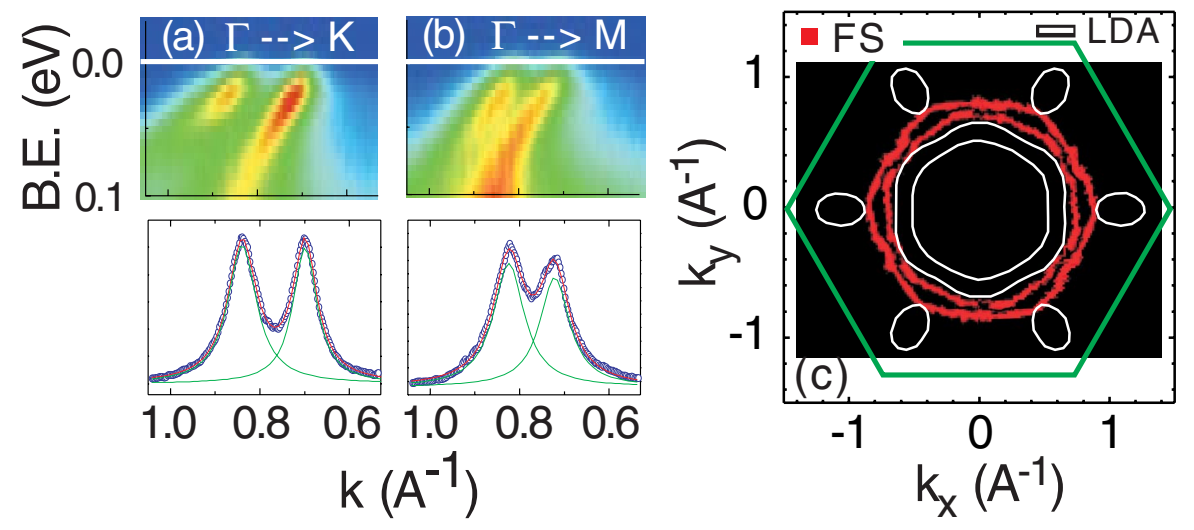

FIG. 4 (color online). (a),(b) A twoband (double) crossing behavior is observed for $x>2 / 3$. Systematics of data are shown for $x=0.8$ where samples exhibit 3D SDW order. The data can be modeled with two Lorentzians. (c) The Fermi surface topology is compared with interlayer splitting in LDA calculation [9]. The observed FS consumes the area of the corner pockets.

pockets are seen (the $e_{g}$ band remains below the Fermi level), and (b) splitting anisotropies are largest along the directions of the absent corner pockets [Fig. 4(c)]. It is known from neutron and $\mathrm{x}$-ray diffraction studies that with increasing $\mathrm{Na}$ content the separation between the two cobalt layers decreases substantially [10,11]. Filling of the Na layer by high $\mathrm{Na}$ density (high doping) leads to stronger bonding between the Co layers, resulting in decreased interplanar ( $c$-axis) distance. Therefore, our observation of bilayer splitting at high doping and its absence at low doping suggest a two- to three-dimensional crossover of the low-lying electronic structure with increasing sodium concentration. The large interlayer splitting observed only at high $x$ suggests that $c$-axis exchange is large, since interlayer hopping and magnetic exchange (exchange interlayer coupling) are directly related [12]. The crucial role of the Na layer (atomic rearrangements) at high doping in determining the magnetic state of the sample is thus consistent with recent NMR findings [13].

The Fermi surface area at high doping does not exactly match the 2D Luttinger count [deviation of data from the dotted line in Fig. 2(d)]. The fact that the Fermi velocity and other band behavior exhibit systematic changes with doping suggests that the deviation cannot be due to doping inhomogeneity. We attribute this deviation in FS area to two factors which were overlooked in earlier studies. The SDW order leads to a canonical doubling of the primitive unit cell, and, since the order is fully $3 \mathrm{D}$, a projected or quasi-2D map of the FS does not necessarily capture the full volume. Thus, the 2D Luttinger count is not applicable to the FS of highly doped cobaltates, where a 3D SDW is observed. This fact and the unresolved bilayer splitting provide clues to the puzzle of the reported FS being larger in previous studies $[4,5]$. To perform a proper Luttinger count, one would need to count the full 3D Fermi surface volume and consider the effect of the full SDW order on the electronic structure.

Based on the vanishing of interlayer coupling in going from high to low doping, we conclude that the electronic structure is largely two-dimensional near the superconducting $\mathrm{Na}$ concentration. We now discuss the possibility of electron-hole excitation induced instabilities with 2D commensurate wave vectors. We note that the 2D FS near $x=1 / 3$ exhibits nearly straight sections perpendicular to the high symmetry directions. At low doping, this distance $2 k_{F}$ is very close to $2 \mathbf{G} / 3$, where $\mathbf{G}$ is the fundamental reciprocal lattice vector (Fig. 3). This is nonorthogonal to the straight sections of the FS. Another instability is that of the $120^{\circ}$ type, which can arise from weak spatial modulations of charge, spin, or orbital densities. Our data show that the electronic system in the vicinity of $x=1 / 3$ is susceptible to such kinematic instabilities. This is due to the fact that the Brillouin zone of such order or fluctuations coincides with the topology of our measured FS. The geometrical construct for $120^{\circ}$ order is shown in real and reciprocal space in Figs. 3(c) and 3(d). The Brillouin zone constructed out of the white hexagons in Fig. 3(d) is superimposed on the measured Fermi surface (white hexagon) presented in Fig. 3(b). The coincidence, including the $k$-space anisotropies, can be noted in Fig. 3(b). For this type of instability, there is no specific single nesting vector and the instability is collective in nature involving all parts of the FS. In order to determine whether or not this instability is unique to $x=0.3$, we have carried out the doping dependence of FS size in the vicinity of $x=0.3$. The uniqueness of this instability $\left(G^{\prime}\right)$ can be seen in Fig. 3(e) - the $G^{\prime}=2 k_{F}$ line intersects the Luttinger line uniquely near $x=0.34$, which is the doping for superconductivity [14].

The doping evolution of electron behavior and lowenergy correlation effects have been theoretically studied in an extended Hubbard model on a triangular lattice [15]. These studies predict strong renormalization of bandwidth (i.e., small Fermi velocity) over the phase diagram. Moreover, a strictly two-dimensional $120^{\circ}$-type collective instability or order, such as $\sqrt{3} \times \sqrt{3}$, near $x=1 / 3$ and $2 / 3$ is predicted. Our results show that only near $x=1 / 3$ is such a two-dimensional instability (with triangular symmetry) allowed with the measured topology of the Fermi surface (Fig. 3). Near $x=2 / 3$, on the other hand, electronic structure is quite three-dimensional. Theory suggests that, if this order is long-range, the quasiparticle spectrum would be gapped at all momenta $(k)$ and the Fermi velocity would exhibit strong renormalization at nearby dopings. In our study, no gap opening is observed in the quasiparticle dispersion [Fig. 2(a)] at any momentum 
TABLE I. Comparison of cobaltates with BCS (Bardeen-Cooper-Schrieffer) and non-BCS cuprate superconductors.

\begin{tabular}{|c|c|c|c|c|c|c|c|}
\hline Class & $T_{c}(\mathrm{~K})$ & $\begin{array}{l}\text { Fermi } \\
\text { velocity } \\
(\mathrm{eVA})\end{array}$ & $\begin{array}{c}\text { Retardation [17] } \\
E_{\text {Fermi }}(W) / \hbar \omega_{\text {Ph }} \text { optic) } \\
\text { (by phonons) }\end{array}$ & $\begin{array}{c}\text { Phase } \\
\text { ordering [17-19] } \\
T_{\theta}(K) \sim n_{2 d(\mathrm{SF})} / m^{*} \\
\end{array}$ & $\begin{array}{c}\text { Mass } \\
\hbar k_{\text {Fermi }} / \nu_{\text {Fermi }} \\
m^{*} / m_{e}\end{array}$ & $\begin{array}{c}\text { Size of the } \\
\text { pair Wfn }(\AA) \\
\sim 0.2\left(\hbar \nu_{\text {Fermi }} / k_{B} T_{c}\right) \\
\end{array}$ & Ref. \\
\hline Cobaltates $(\mathrm{NaCoO})$ & 5 & $0.37 \pm 0.1$ & $<4$ & $<\mathbf{2 0}$ & $>15$ & $\sim 200$ & Present work \\
\hline$p$ cuprates $(\mathrm{LaSrCuO})$ & 38 & 1.8 & 8 & 54 & 2 (nodal) & $\sim 100$ & {$[17,20]$} \\
\hline$n$ cuprates $(\mathrm{NdCeCuO})$ & 21 & 2.0 & 9 & 130 & 2.4 (nodal) & $\sim 210$ & {$[17,20]$} \\
\hline $\mathrm{MgB}_{2}$ & 39 & $2-7$ & $10^{2}$ & $10^{3}$ & $x$ & $x$ & [21] \\
\hline Lead $(\mathrm{Pb})$ & 7.2 & 10.4 & $10^{3}$ & $10^{5}$ & 1.8 & $10^{4}$ & {$[17,22]$} \\
\hline
\end{tabular}

at this doping down to the base temperature of the experiment. However, the Fermi velocity is seen to be suppressed over a range of doping [Fig. 2(e)]. Therefore, our results suggest a strongly fluctuating character of this hidden collective instability [14].

The strong correlation models have further been considered in connection with superconductivity $[15,16]$. These studies suggest that a kinematic or hidden FS instability with strong fluctuations can lead to unconventional superconductivity with an $f$-wave order parameter, even within a Fermi-liquid quasiparticlelike picture. The quasiparticle parameters related to the cobalt-derived states with triangular symmetry are, therefore, of interest for theory. Based on our data, we estimate the parameters in cobaltates presented in Table I. For comparison, we also quote similar parameters obtained on conventional materials as well as the unusual materials classes. For use in Table I, the Fermi energy of cobaltates is estimated to be on the order of the occupied bandwidth (following the approximation in Ref. [17]), which is taken from our data in Fig. 2(f). At the order-of-magnitude level, our results show that the cobaltate exhibits a lack of a retardation effect due to its small Fermi energy, possesses a small phase ordering (kinetic energy) scale due to its large effective mass, and exhibits a relatively short coherence length (pair wave function) due to its small Fermi velocity. All these characteristics can be traced to the strong renormalization of the Fermi velocity, which, in cobaltates, is about a factor of 5 smaller than it is in the high $T_{c}$ cuprates. Given such character for the low-lying states and the $k$-space kinematics we observe, it is unlikely that a conventional superconducting mechanism is at play (see Table I).

In conclusion, fine details of the low-energy states in $(\mathrm{Na} / \mathrm{K})_{x} \mathrm{CoO}_{2}$ are resolved due to the high crystalline quality of materials. A splitting of the single-particle band signalling bilayer coupling is observed in the highdoping regime which accounts for the observed threedimensional magnetism. In the low-doping regime, in contrast, the splitting disappears, leading to the twodimensional character of triangularly correlated electron motion. Our results suggest that the small Fermi velocity and the unique two-dimensional symmetry of kinematic instabilities distinguish cobaltates from most other oxide superconductors and clearly so from conventional BCS superconductors. The quantitative details (Table I) of the low-lying states in our studies provide important guides to develop a comprehensive theory of cobaltates over a large part of the phase diagram.

We gratefully acknowledge P. W. Anderson, D. A. Huse, S. A. Kivelson, P. A. Lee, N.P. Ong, S. Shastry, and S. Sondhi for discussions. This work is partially supported through the NSFMRSEC (DMR-0213706), DOE Grants No. DE-FG02-05ER46200 and No. DE-FG02-98ER45706, and NSFC (Grant No. 10574158). ALS is supported by DOE/BES-DEAC03-76SF00098.

*Electronic address: mzhasan@princeton.edu

[1] K. Takada et al., Nature (London) 422, 53 (2003).

[2] I. Terasaki et al., Phys. Rev. B 56, R12 685 (1997).

[3] N. P. Ong and R. J. Cava, Science 305, 52 (2004).

[4] M.Z. Hasan et al., Phys. Rev. Lett. 92, 246402 (2004).

[5] H. B. Yang et al., Phys. Rev. Lett. 95, 146401 (2005).

[6] A. Kanigel et al., Phys. Rev. Lett. 92, 257007 (2004).

[7] Y.-D. Chuang et al., Phys. Rev. Lett. 87, 117002 (2001).

[8] D. L. Feng et al., Phys. Rev. Lett. 86, 5550 (2001).

[9] D. J. Singh, Phys. Rev. B 61, 13397 (2000).

[10] Q. Huang et al., Phys. Rev. B 70, 184110 (2004).

[11] M. Foo et al., Phys. Rev. Lett. 92, 247001 (2004).

[12] S. P. Bayrakci et al., Phys. Rev. Lett. 94, 157205 (2005).

[13] C. de Vaulx et al., Phys. Rev. Lett. 95, 186405 (2005).

[14] We note a natural enlargement of the central FS at $x=$ $1 / 3$ in our data (Fig. 3) with respect to LDA predictions given the absence of corner FS pockets (Refs. [4,5]) and the constraint of the Luttinger theorem. Remarkably, our results suggest that both the suppression of corner pockets and the enhanced susceptibility to $120^{\circ}$-type order are natural consequences of long-range Coulomb interactions.

[15] O. Motrunich and P. A. Lee, Phys. Rev. B 70, 024514 (2004).

[16] Y. Tanaka et al., cond-mat/0311266.

[17] E. W. Carlson et al., in The Physics of Conventional and Unconventional Superconductors, edited by K. H. Bennemann and J. B. Ketterson (Springer-Verlag, Berlin, 2002).

[18] V. Emery and S. A. Kivelson, Nature (London) 374, 434 (1995).

[19] Since the superfluid density is not known, we have used the Fermi sea to calculate the phase ordering scale.

[20] A. Damascelli et al., Rev. Mod. Phys. 75, 473 (2003); A. Kaminski et al., Phys. Rev. B 71, 014517 (2005).

[21] H. Uchiyama et al., Phys. Rev. Lett. 88, 157002 (2002).

[22] K. Horn et al., Phys. Rev. B 30, 1711 (1984). 